\title{
EFECTO DE LA DENSIDAD DE SIEMBRA Y EL AMBIENTE DE CULTIVO SOBRE EL RENDIMIENTO Y LA CALIDAD DE TALLOS DE LOS CULTIVARES DE ROMERO (Rosmarinus officinalis L.) CRESPO E ISRAELÍ
}

Fecha de recepción: 11 de agosto de 2013 • Fecha de aceptación: 3 de septiembre de 2013

\section{EFFECT OF PLANTING DENSITY AND CROP ENVIRONMENT ON YIELD AND QUALITY OF ROSEMARY CULTIVARS CRESPO AND ISRAELI.}

María Elena Cortés-Rojas ${ }^{1}$ Fabio Andrés Cañón Angarita² $•$ Daniel Rodríguez Caicedo³ $\bullet$ María Mercedes Pérez Trujillo ${ }^{3,4}$

\section{RESUMEN}

El romero es una planta aromática reconocida por su alta capacidad antioxidante y sus numerosos usos y propiedades medicinales. Sin embargo, la información relacionada al efecto de los factores agroambientales sobre el cultivo de romero no está suficientemente documentada. El objetivo de este estudio fue evaluar la productividad de los cultivares de romero Crespo e Israelí sembrados bajo tres densidades de siembra (30 x 30,40 × 40 y 60 × $60 \mathrm{~cm}$ de espaciamiento entre hileras y entre plantas), y cultivados tanto en campo abierto como bajo invernadero. Se utilizó un diseño de bloques completos al azar con cuatro repeticiones. Las variables evaluadas fueron biomasa fresca y seca de los tallos cosechados durante tres cortes, así como su clasificación en las categorías de calidad nacional, exportación y extra. Los rendimientos más altos para la mayoría de las variables fueron obtenidos al disponer un mayor número de plantas por área y en el cultivo sembrado en campo abierto, excepto para el peso fresco de los tallos clasificados en la categoría extra donde se destacó el invernadero y especialmente el cultivar Israelí. Respecto al comportamiento productivo de los cultivares, el Crespo presentó una mayor producción de tallos categoría nacional, mientras que el Israelí fue superior en la categoría exportación.

Palabras clave: plantas aromáticas, cultivos protegidos, rendimiento, crecimiento.

1. Bióloga, Grupo de Agrobiología de especies vegetales promisorias de clima frío, Facultad de Ciencias Básicas y Aplicadas, Universidad Militar Nueva Granada.

2. Estudiante de pregrado de Biología Aplicada, Grupo de Agrobiología de especies vegetales promisorias de clima frío, Facultad de Ciencias Básicas y Aplicadas, Universidad Militar Nueva Granada.

3. Docente Investigador, Grupo de Agrobiología de especies vegetales promisorias de clima frío, Facultad de Ciencias Básicas y Aplicadas, Universidad Militar Nueva Granada.

4. Autor para correspondencia: agrobiologia@unimilitar.edu.co 


\section{ABSTRACT}

Rosemary is an aromatic plant, well known for its antioxidant capacity and its numerous uses and medical properties. Agronomical factors affecting rosemary production and its development in different conditions has been little studied.

Thus, this work aims to evaluate the yield of the rosemary cultivars Crespo and Israeli grown in three planting densities ( 30 × 30, 40 × 40 y 60 × 60 cm plant to plant and inter-row distance) under open field and greenhouse conditions. The experimental design was a randomized complete block with four replications. In order to study the effects of planting density and agronomical conditions, yields of dry and fresh weight and classification of shoots in the quality classes nacional, exportacion and extra were measured.

Results showed that the highest improvement in yield was obtainable by a major crop density and in open field conditions except in the extra quality class were the highest productivity was obtained under greenhouse conditions and specially in the cultivar Israeli. The highest yield in the nacional quality class was obtained in the cultivar Crespo and the cultivar Israeli provided the maximum yield in the exportación quality class.

Keywords: aromatic plants, crop protection, productivity, growth.

\section{INTRODUCCIÓN}

El consumo de plantas aromáticas y medicinales está asociado con hábitos de vida más saludables al ser reconocidas por sus aportes de nutrientes y por las propiedades sobre el tratamiento de enfermedades y dolencias (Viuda-Martos et al., 2007; Moreno et al., 2012). Esto ha generado que en los últimos años la demanda mundial por estos productos sea creciente, sumado a la diversidad racial y a las preferencias culturales por las comidas condimentadas (Cracker, 2007; Souza et al., 2008; Xiao et al., 2008).

El romero pertenece a la familia Lamiaceae y es originario del Mediterráneo (Marin et al., 2006; Maganga, 2004). Se destaca dentro de las plantas aromáticas y medicinales como una de las de mayor actividad antioxidante (Peng et al., 2005; Luis et al., 2007; Hernández-Hernández et al., 2009; Moreno et al., 2012). Ha sido empleado desde la antigüedad como condimento y para el tratamiento de diversas enfermedades (Al-Sereiti et al., 1999). En la actualidad muchas investigaciones se centran en el estudio de sus ingredientes activos para ser empleados en la industria cosmética, alimenticia y farmacéutica. El romero es usado para problemas del sistema nervioso, circulatorio, para estimular el crecimiento del cabello e incluso como insecticida y herbicida en agricultura (Al-Sereiti et al., 1999; Katerinopoulos et al., 2005; Ávila-Sosa et al., 2011). Así mismo, se ha reportado su potencial como follaje en la elaboración de bouquets (Gutierrez, 2007). Se comercializa en fresco, seco o como aceite esencial en el que se pueden encontrar compuestos como flavonoides, diterpenos, esteroides y triterpenos que le confieren propiedades antimicrobiales y antifúngicas entre otras (Yanishlieva-Maslarova y Heinoren, 2001; Porte y de Oliveira, 2001; Sasikumar, 2004; Genena et al., 2008; Socaci et al., 2008). 
En cuanto al cultivo de romero, la literatura cita que esta especie puede ser plantada a diferentes densidades de siembra, que van desde $25 \mathrm{~cm}$ hasta 50 cm entre plantas (Sasikumar 2004; Bareño 2006; Alarcón 2011), sin embargo los reportes formales que se enfoquen a la evaluación de diferentes densidades de siembra sobre el rendimiento del cultivo, son escasos. En general, es bien conocido que la densidad de siembra es el principal factor relacionado con los parámetros de crecimiento y rendimiento de los cultivos (Baloch et al., 2002; Chang et al., 2008) y que el uso adecuado de los recursos de agua, luz, absorción de nutrientes y exposición a la radiación solar, se relacionan con una densidad de plantación adecuada (Sadeghi et al., 2009; Khorshidi et al., 2009).

Al respecto, Naghdi et al. (2004) y Al-Rammamneh (2009) evaluaron el efecto de tres densidades de siembra $(15,30$ y $45 \mathrm{~cm}$ ) sobre la producción de materia fresca y seca en plantas de tomillo. Sus resultados coinciden en que la mayor altura de tallos, biomasa fresca y seca se registró en la densidad de $15 \mathrm{~cm}$. Al sembrar un mayor número de plantas por área, se incrementa la cobertura vegetal y el uso de luz, lo que se refleja en la acumulación de biomasa. El estudio de Naghdi et al. (2004), evaluó también el rendimiento del aceite esencial de tomillo bajo las diferentes densidades y demostró que no hubo efecto de la densidad de siembra sobre este parámetro, sin embargo, la mayor disponibilidad de materia fresca y seca por unidad de área en la densidad de $15 \mathrm{~cm}$ favoreció un rendimiento superior del aceite esencial.

En un estudio realizado por Ferreira et al. (2013) en otra especie de planta aromática, Occimum gratissimum, evaluaron diferentes intensidades lumínicas sobre el crecimiento, rendimiento de aceite esencial y variación en la densidad de tricomas en las hojas. Encontraron una relación lineal entre la cantidad de luz y la producción de biomasa; aunque la densidad de tricomas fue mayor en los tratamientos con mayor luminosidad, no hubo una relación entre la intensidad de luz y la producción de aceites esenciales. Con relación al rendimiento del aceite esencial, estos resultados coinciden con los reportados por Naghdi et al. (2004), al presentar los mayores valores debido al aumento en la biomasa.

La luz interceptada por las hojas depende de factores como la densidad de siembra, la orientación del cultivo y la arquitectura de la planta (Li et al., 2000) y tiene un rol clave en la biosíntesis de metabolitos, por tanto, es determinante en la producción y composición de aceites esenciales en plantas aromáticas y medicinales (Li y Cracker, 1996; Chang et al., 2008; Gomes et al., 2009). Particularmente en los sistemas de cultivo bajo cubierta como los invernaderos, la cantidad de luz recibida es menor en relación a los cultivos a campo abierto, ya que depende de condiciones como el tipo de plástico, espesor, limpieza, calibre, forma de la cubierta, estructura, entre otros, lo que genera, junto a otros factores ambientales como temperatura y humedad relativa, microclimas diferentes (Ibrahim y Jaafar, 2011, Espí, 2012).

El presente trabajo buscó evaluar el rendimiento del cultivo de romero, expresado como el peso fresco y seco de los tallos cortados, así como la calidad de los mismos según su clasificación en categorías determinadas por su longitud, en dos cultivares de romero, Crespo e Israelí, plantados tanto en campo abierto como bajo invernadero a tres densidades de siembra (30, 40 y $60 \mathrm{~cm}$ de espaciamiento entre plantas).

\section{MATERIALES Y MÉTODOS}

La investigación se desarrolló en el campo experimental de la Universidad Militar Nueva Granada ubicado a $4^{\circ} 56^{\prime} \mathrm{N}, 74^{\circ} 00^{\prime} \mathrm{O}$ y $2562 \mathrm{msnm}$, en el municipio de Cajicá (Cundinamarca, Colombia). La temperatura media, máxima y mínima del aire que 
se presentó en el invernadero fue en promedio de 16.4, 28.7 y $9.7^{\circ} \mathrm{C}$, mientras que en campo abierto fue de $13.5,20.5$ y $8.8^{\circ} \mathrm{C}$. La humedad relativa media, máxima y mínima promedio fue de 74, 91 y $44 \%$ en el invernadero y de 82,99 y $51 \%$ en el campo abierto.

Las plantas empleadas para el establecimiento de los cultivos fueron propagadas a partir de esquejes obtenidos para cada cultivar de una plantación comercial ubicada en El Rosal (Cundinamarca), los cuales, luego del proceso de enraizamiento, fueron trasplantados. En campo abierto las plantas fueron sembradas en el terreno natural en un suelo alfisol (Fig. 1 A). En invernadero, el cual tenía una estructura metálica y cubierta plástica transparente (Fig. 1B), las plantas fueron dispuestas en camas contenidas con sustrato conformado por tierra negra (suelo andisol), cascarilla de arroz tostada y compost en proporción 2:1:1.

Tanto en invernadero como en campo abierto el área experimental fue de $202 \mathrm{~m}^{2}$. En cada ambiente de cultivo se instalaron 6 camas de $30 \mathrm{~m}$ de longitud, $1.10 \mathrm{~m}$ de ancho y $0.30 \mathrm{~m}$ de alto, cubiertas con acolchado plástico de color negro y dotadas con riego por goteo (Fig. 1). Las camas fueron divididas longitudinalmente en cuatro parcelas.

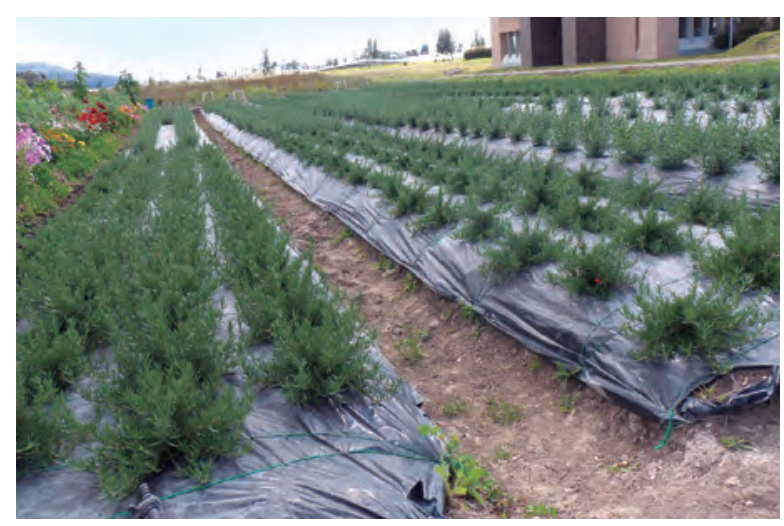

(A)
Se utilizó un diseño de bloques completos al azar con arreglo factorial de $2 \times 3 \times 2$, siendo el primer factor el cultivar de romero (Israelí o Crespo), el segundo la densidad de siembra (30x30, 40x40 o $60 \times 60 \mathrm{~cm}$ de espaciamiento entre plantas) y el tercer factor el ambiente de cultivo (invernadero y campo abierto). Se evaluaron 12 tratamientos correspondientes a las combinaciones de los dos cultivares, las tres densidades de siembra y los dos ambientes. Se trabajaron 4 bloques, los cuales consistieron en las 4 parcelas en las que fueron divididas las camas.

Semanalmente se registró la longitud de 5 tallos marcados por tratamiento en cada bloque y se programaron las cosechas cuando el $90 \%$ de los tallos alcanzó una longitud igual o superior a $15 \mathrm{~cm}$. A lo largo del experimento se realizaron 3 cortes o cosechas: a los 120, 212 y y 314 días después de la siembra (dds).

En cada corte se evaluaron como variables de rendimiento la biomasa fresca (PF) y la biomasa seca (PS) de la totalidad de los tallos cosechados por unidad de área. Para la obtención de la biomasa seca el material fue sometido a un proceso de secado empleando un horno universal a $70{ }^{\circ} \mathrm{C}$ por un periodo de 72 horas. Como variable de calidad se determinó la biomasa fresca de los tallos clasificados en cada

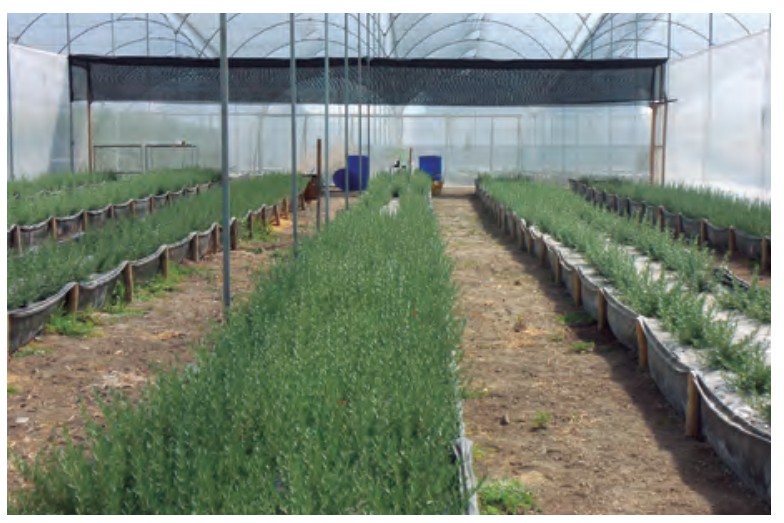

Figura 1. A. Vista del área experimental del cultivo de romero Rosmarinus officinalis sembrado en campo abierto (A) y bajo invernadero (B). Fotos: M.E. Cortés (2014). 
una de las categorías comerciales de calidad, las cuales se basaron en su longitud: nacional (menos a $14.9 \mathrm{~cm}$ ), extra (entre 15-19.9cm) y exportación (mayor a $20 \mathrm{~cm}$ ).

Los datos que se procesaron para el análisis de cada variable correspondieron a lo acumulado durante las tres primeras cosechas, las cuales se obtuvieron durante los 10 primeros meses de edad del cultivo. Los resultados obtenidos fueron sometidos a análisis combinado de varianza y las comparaciones múltiples fueron realizadas con pruebas de Tukey. El software estadístico utilizado fue R v. 2.15.2, en su versión libre.

\section{RESULTADOS}

Los resultados del análisis de varianza indicaron que el ambiente, la densidad de siembra y el cultivar, tuvieron un efecto significativo sobre el rendimiento del cultivo y la calidad del producto cosechado. Así mismo, se presentaron interacciones entre algunos de estos factores para las variables de biomasa seca y de biomasa fresca de tallos de categoría nacional y exportación (Tabla 1).

Independiente del cultivar de romero y de la densidad de siembra empleada, el mayor rendimiento del cultivo, expresado mediante la biomasa fresca, fue obtenido en el campo abierto con $1395 \mathrm{~g} / \mathrm{m}^{2}$, superando estadísticamente los $1244 \mathrm{~g} / \mathrm{m}^{2}$ que se presentaron en el invernadero (Tabla 1 y Figura 2A). En cuanto al efecto del espaciamiento entre plantas, la mayor cantidad de biomasa fresca cosechada se registró para la densidad de $30 \times 30 \mathrm{~cm}$, siendo superior en un $30 \%$ y $56 \%$ a la obtenida en las densidades de 40 × 40 y $60 \times 60 \mathrm{~cm}$, respectivamente (Figura 2B). Por su parte, no se encontró diferencia estadística alguna entre los cultivares respecto a su rendimiento en peso fresco (Figura 2C).

Tabla 1. Valores de $\operatorname{Pr}(>F)$ obtenidos en el ANOVA, al analizar el efecto del cultivar, la densidad de siembra, el ambiente de cultivo y sus interacciones, sobre las variables de rendimiento del cultivo de romero y la calidad de los tallos cosechados 1.

\begin{tabular}{|c|c|c|c|c|c|c|}
\hline & df & $\begin{array}{c}\text { PF } \\
\left(g / m^{2}\right)\end{array}$ & $\begin{array}{c}\text { PS } \\
\left(\mathrm{g} / \mathrm{m}^{2}\right)\end{array}$ & $\begin{array}{c}\text { Nal } \\
\left(\mathrm{g} / \mathrm{m}^{2}\right)\end{array}$ & $\begin{array}{c}\text { Exp } \\
\left(g / m^{2}\right)\end{array}$ & $\begin{array}{c}\text { Extra } \\
\left(\mathrm{g} / \mathrm{m}^{2}\right)\end{array}$ \\
\hline Cultivar & 1 & 0.603 & 0.103 & $<0.001$ & 0.44203 & 0.0267 \\
\hline Densidad & 2 & $<0.001$ & $<0.001$ & $<0.001$ & $<0.001$ & $<0.001$ \\
\hline Ambiente & 1 & 0.005 & $<0.001$ & $<0.001$ & $<0.001$ & $<0.001$ \\
\hline Cultivar: Densidad & 2 & 0.470 & 0.358 & 0.010 & 0.39813 & 0.123 \\
\hline Cultivar: Ambiente & 1 & 0.096 & 0.011 & 0.363 & 0.04619 & 0.372 \\
\hline Densidad: Ambiente & 2 & 0.158 & 0.018 & 0.695 & 0.003 & 0.966 \\
\hline Ambiente: Bloque & 6 & 0.009 & 0.026 & 0.379 & 0.179 & 0.109 \\
\hline Cultivar: Densidad: Ambiente & 2 & 0.409 & 0.146 & 0.126 & 0.836 & 0.784 \\
\hline Residuales & 30 & & & & & \\
\hline
\end{tabular}

${ }^{1}$ En negrilla $\mathrm{P}(>\mathrm{F})<0.05,0.01$ y 0.001 , respectivamente. PF: biomasa fresca; PS: biomasa seca;

Nal: biomasa fresca de tallos de calidad nacional; Exp: biomasa fresca de tallos de calidad exportación; Extra: biomasa fresca de tallos calidad extra. 


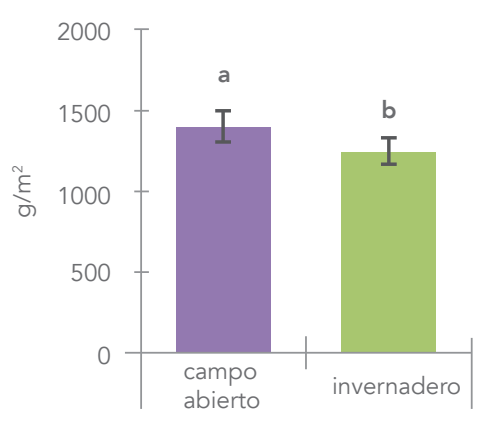

(A)
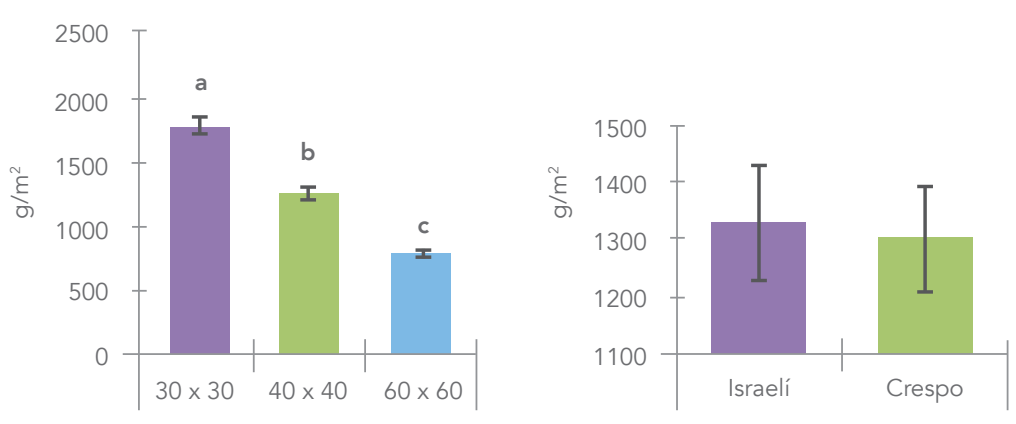

(B)

Figura 2. Biomasa fresca acumulada en campo abierto e invernadero (A); bajo tres densidades de siembra (B) y entre cultivares (C).

Al analizar el rendimiento del cultivo expresado a través de la biomasa seca de los tallos cosechados, también se encontró que los factores densidad de siembra y ambiente de cultivo influyeron significativamente y de manera independiente sobre esta variable (Tabla 1). El mayor rendimiento del romero se obtuvo a la mayor densidad de siembra (Figura 3A), y en el ambiente de campo abierto ( $F i$ gura 3B), mientras que no se apreció una diferencia entre los cultivares (Figura 3C). Sin embargo, si se encontró que los cultivares respondieron de manera diferencial ante el ambiente de cultivo (Tabla 1), siendo mayor la producción de biomasa seca en el romero Israelí sembrado en campo abierto (Figura 3D). Así mismo, se presentó interacción entre los factores densidad de siembra y el ambiente de cultivo (Tabla 1), destacándose particularmente el mayor rendimiento obtenido en las plantas de romero sembradas a una distancia de siembra de $30 \times 30$ $\mathrm{cm}$ en campo abierto (Figura 3E).

Respecto a la calidad de los tallos de romero, se encontró para la categoría nacional una influencia significativa tanto del cultivar, la densidad de siembra y el ambiente de cultivo, así como interacción entre el cultivar y la densidad (Tabla 1). El cultivar Crespo tuvo una producción significativamente mayor de tallos categoría nacional frente al Israelí (275.9 y $210.3 \mathrm{~g} / \mathrm{m}^{2}$ respectivamente) (Figura $4 \mathrm{~A}$ ). Respecto a las densidades evaluadas, la mayor producción de tallos de esta categoría se registró al disponer plantas con un espaciamiento de $30 \times 30 \mathrm{~cm}$ (Figura 4B) y en el campo abierto, con un promedio de $310.9 \mathrm{~g} /$ $\mathrm{m}^{2}$ (Figura 4C). Particularmente, se destacó el romero Crespo en la densidad de $30 \times 30 \mathrm{~cm}$ (Figura 4D).

En la categoría exportación, se evidenciaron efectos significativos tanto del ambiente de cultivo como de la densidad (Tabla 1), independientes del cultivar. La mayor biomasa fresca de tallos clasificados en esta calidad se obtuvo en el campo abierto (Figura 5A) y a la densidad de $30 \times 30 \mathrm{~cm}$ (Figura $5 B)$. Se evidenció la influencia combinada de los factores Densidad:Ambiente (Tabla 1), ya que la mejor respuesta se obtuvo al sembrar el romero a la densidad de siembra de $30 \times 30 \mathrm{~cm}$ en campo abierto (Figura 5D).

Aunque no se presentaron diferencias entre los cultivares (Figura 5C), si se encontró una relación entre el cultivar y el ambiente, ya que el romero Israelí se destacó en la producción de tallos calidad exportación en campo abierto, mientras que en el invernadero fue el Crespo. Dado que estas diferencias estuvieron muy cercanas al límite del nivel de significancia en el ANOVA (Tabla 1), la prueba de Tukey no alcanzó a reflejarlas (Figura 5E).

La biomasa fresca de los tallos de mayor longitud, clasificados en la categoría extra, difirió 


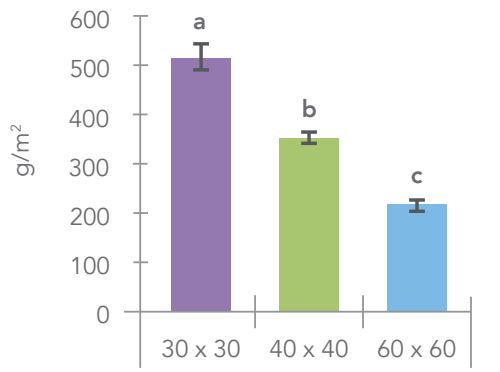

(A)

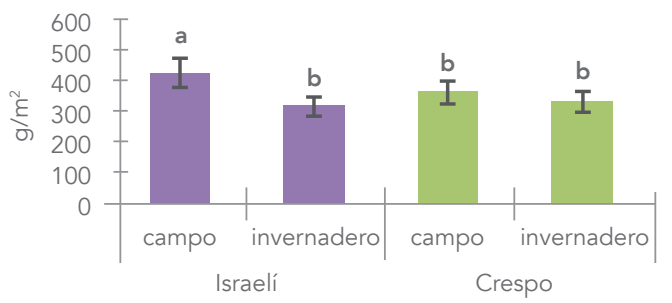

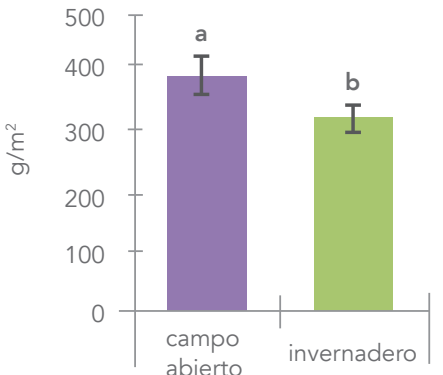

abierto invernadero

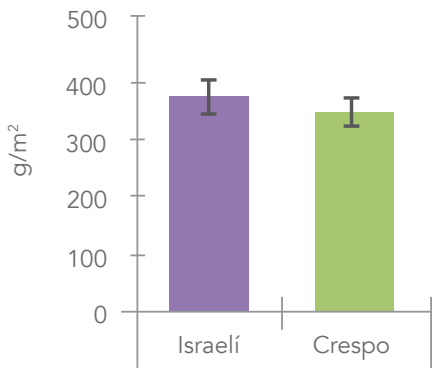

(B)

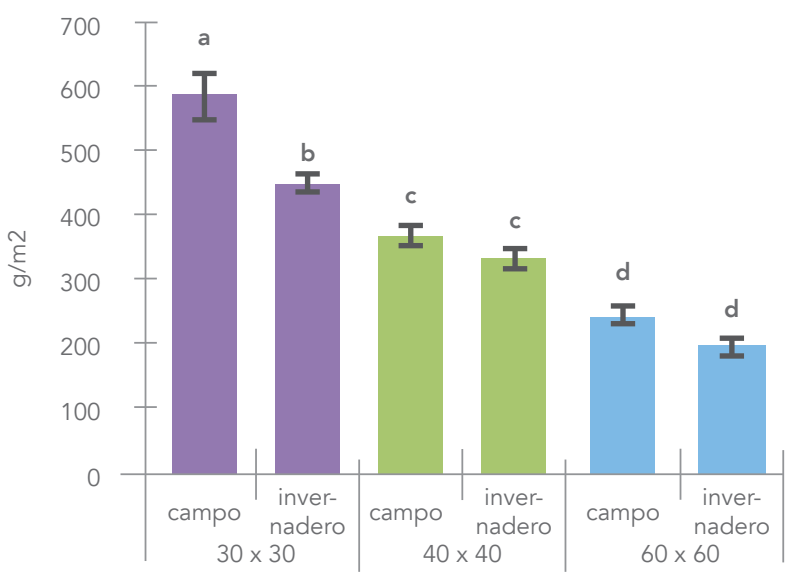

(D)

Figura 3. Biomasa seca en función de las densidades de siembra (A); en función de los ambientes de cultivo (B); entre cultivares (C); interacción ambiente por cultivar (D) e interacción densidad por ambiente (E).

significativamente en función del cultivar, de la densidad de siembra y del ambiente de cultivo (Tabla 1). Esta fue mayor a la densidad de siembra más alta (30 x $30 \mathrm{~cm}$ ) (Figura 6A), y para el romero Israelí frente al Crespo (Figura 6B). Sin embargo, contrario a lo ocurrido con las otras variables, la mayor la producción de estos tallos fue en invernadero $\left(705.4 \mathrm{~g} / \mathrm{m}^{2}\right)$ en comparación al campo abierto $\left(465.9 \mathrm{~g} / \mathrm{m}^{2}\right)$ (Figura 6C).

\section{DISCUSIÓN}

Con relación a la densidad de siembra, en general los mayores rendimientos del cultivo del romero así como la calidad de los tallos cosechados, fueron obtenidos al disponer un mayor número de plantas por unidad de área. Estos resultados concuerdan con lo reportado por Soto et al. (2003), quienes evaluaron el rendimiento de la planta medicinal Cymbopogon citratus Stapf. sembrada bajo diferentes densidades de siembra y en el que los rendimientos más altos por hectárea los obtuvieron en las mayores densidades de siembra. Lo anterior coincide también con los resultados obtenidos por Al-Ramamneh (2009) en tomiIlo, donde la mayor acumulación de biomasa fresca y seca se presentó al sembrar las plantas a una distancia de $15 \mathrm{~cm}$, siendo la mayor densidad evaluada. 
Las plantas responden a la proximidad de otras plantas por medio de cambios morfológicos y fisiológicos. En el caso de una mayor densidad de siembra hay un aumento en la cantidad de tallos por unidad de área, lo que produce por lo general mayor sombreamiento. Bajo estas condiciones, se activan fotorreceptores (fitocromos) que por medio de un proceso hormonal (giberelinas y auxinas), promueven la elongación de tallos como respuesta adaptativa dirigida a incrementar la cantidad de luz interceptada. Esto a su vez, tiene un impacto sobre la acumulación de biomasa, al translocar los fotoasimilados a los tallos (Ballaré et al., 1991; Ballaré et al., 1994; Dudley y Schmitt, 1995; Pierik et al., 2004; Lambers et al., 2008, Taiz y Zeiger, 2010), lo que podría explicar los resultados en cuanto a mayor acumulación de biomasa fresca y seca para el romero, independiente del cultivar y del ambiente de cultivo, cuando fue sembrado a la densidad de $30 \times 30 \mathrm{~cm}$.

Así mismo, se ha reportado que el sombreamiento influye también en el patrón de ramificación y arquitectura de las plantas. Es así que hay una notable disminución del número de ramas cuando la disponibilidad de luz es menor, y estas adoptan una posición más vertical en las plantas (Aphalo y Ballaré, 1995; Morelli y Ruberti 2002). Esto fue evidente al comparar las diferentes densidades de siembra, en las que las plantas a menor densidad, presentaron un patrón de crecimiento más ramificado que cuando se sembraron a una mayor densidad.

En cuanto a los cultivares de romero evaluados, solo se presentaron diferencias en las variables de biomasa seca y de biomasa fresca de tallos de las categorías nacional y extra. Teniendo en cuenta que el valor comercial del romero son sus tallos, medido en acumulación de biomasa y en longitud, especialmente para el mercado en fresco, los mejores resultados se obtuvieron en el material Israelí.

Esto se puede atribuir a las diferencias en el hábito de crecimiento de los cultivares. El romero se

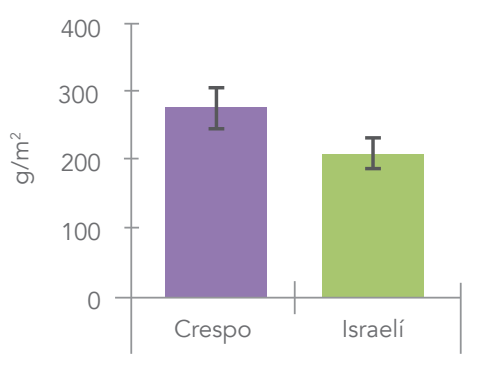

(A)

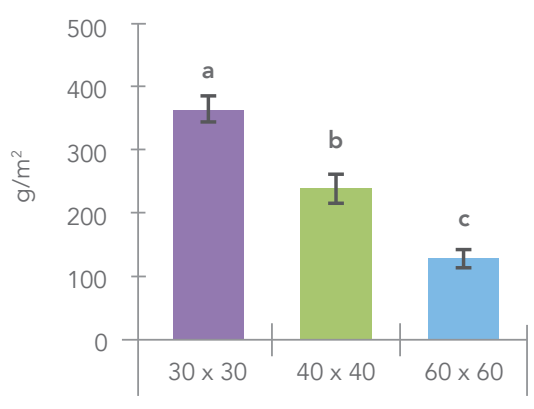

(B)
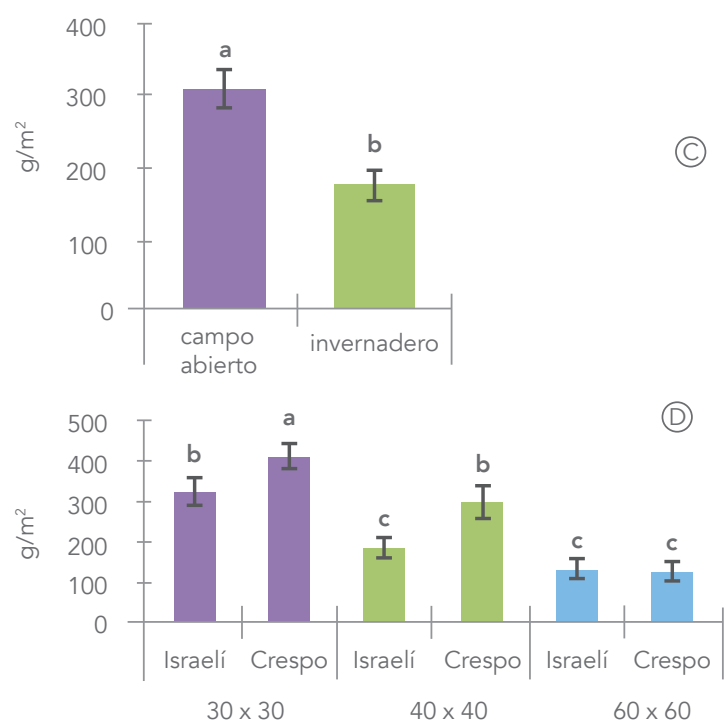

Figura 4. Biomasa fresca de los tallos clasificados en la categoría nacional entre cultivares $(A)$; en función de las densidades de siembra (B); en función de los ambientes de cultivo (C) y en la interacción cultivar y densidad (D).

caracteriza por ser polimórfico, siendo el hábito de crecimiento una de las características que se utiliza para la identificación de los materiales presentándose erecto, compacto o rastrero (Roselló et al., 2006; Zaouali et al., 2010). Es así, que el Israelí se 

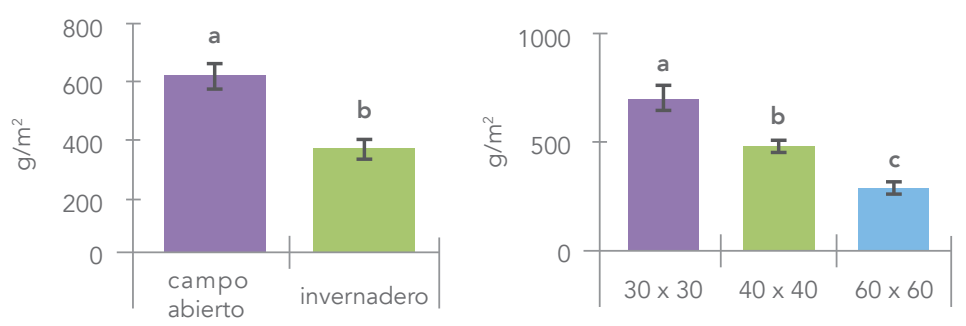

(A)

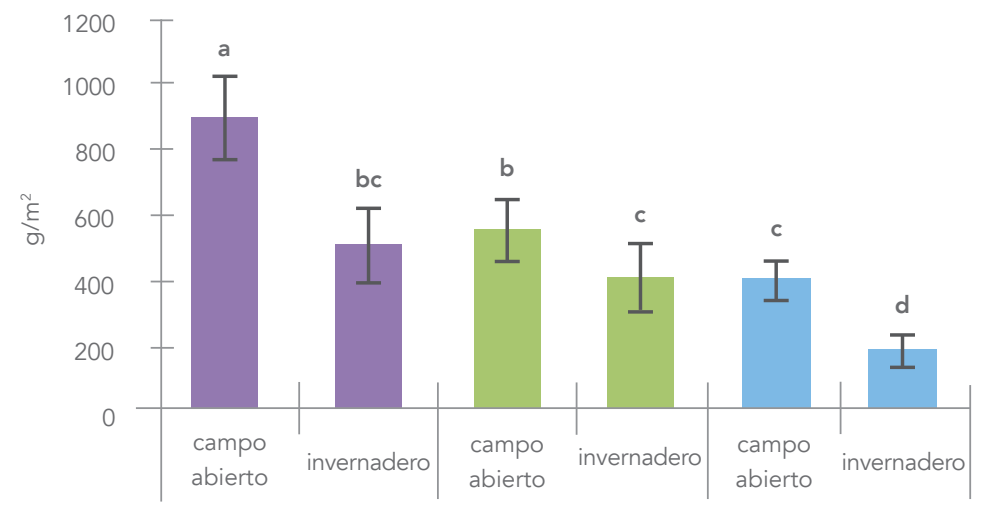

$40 \times 40$

$60 \times 60$

(B)
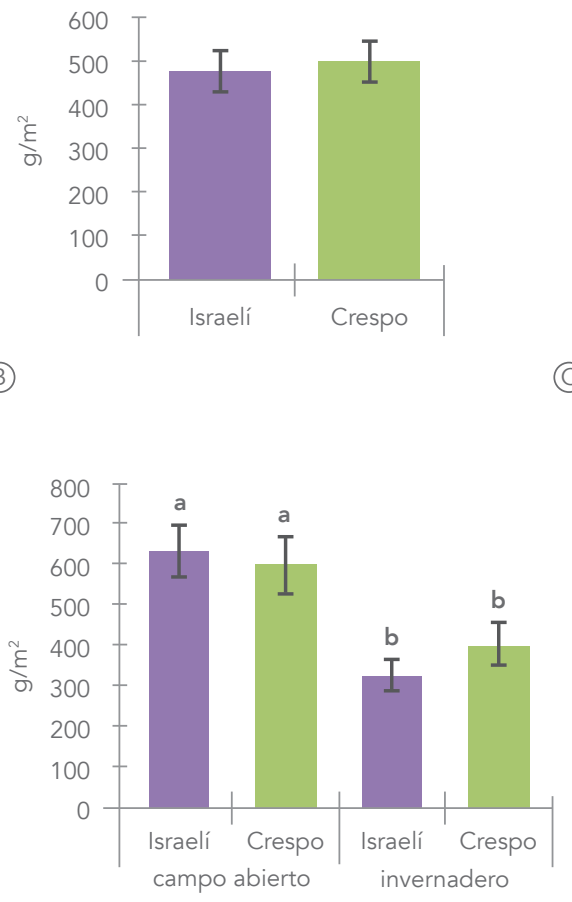

(D)

Figura 5. Producción de tallos de romero clasificados en la categoría exportación en función de los ambientes de cultivo (A); en función de las densidades de siembra (B); entre cultivares (C); en la interacción densidad por ambiente (D) y en la interacción densidad por ambiente (E)

caracteriza por tener un hábito de crecimiento más erecto y menos ramificado, entrenudos más largos y tallos con características de madera blanda, mientras que el Crespo por su parte, es más ramificado, de hábito más rastrero, presenta entrenudos más cortos y tallos de apariencia semileñosa.

En la mayoría de los casos donde se presentaron diferencias entre los ambientes de cultivo probados, el campo abierto registró los mayores valores de rendimiento y calidad, excepto para la categoría extra que fue mayor en el invernadero. Estos resultados pueden estar relacionados con las características propias de los ambientes de cultivo, las cuales involucraron el tipo de suelo o de sustrato y las condiciones meteorológicas de los dos sistemas. Con relación a la temperatura, el romero es una planta xerofítica, adaptada a condiciones de temperatura extrema (Bonilla y Martínez, 2010), sin embargo su rango óptimo para el desarrollo se encuentra entre 19 y $25^{\circ} \mathrm{C}$, con máxima de $26^{\circ} \mathrm{C}$ (González- Michel, 2013). En este experimento, la temperatura diaria promedio en campo abierto fue de $13.5^{\circ} \mathrm{C}$ y en invernadero de $16.4^{\circ} \mathrm{C}$ siendo $2.9^{\circ} \mathrm{C}$ mayor. A pesar que la temperatura media registrada en cada uno de los ambientes se encuentra cercana al rango óptimo de desarrollo del cultivo, especialmente en el invernadero, las temperaturas máximas y mínimas reflejan las fluctuaciones que se presentaron dentro de cada uno de los ambientes. Al comparar la temperatura mínima registrada en 


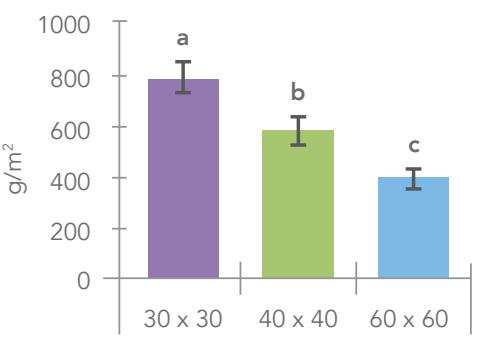

(A)
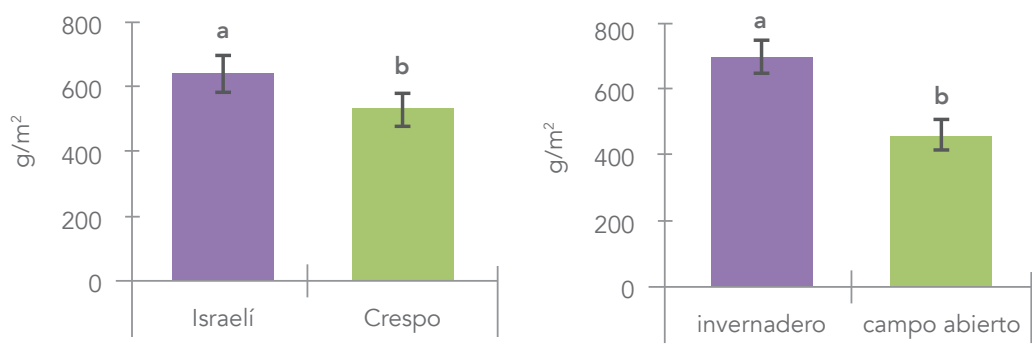

(B)

(C)

Figura 6. Producción de tallos de romero clasificados en la categoría extra en función de la densidad de siembra (A); entre cultivares (B) y en función de los ambientes de cultivo (C)

campo abierto, de $8.8^{\circ} \mathrm{C}$ frente a $9.7^{\circ} \mathrm{C}$ en invernadero se tiene una diferencia de $0.8^{\circ} \mathrm{C}$. Sin embargo, al comparar las temperaturas máximas alcanzadas en los dos ambientes, $20.5^{\circ} \mathrm{C}$ en campo abierto y $28.8^{\circ} \mathrm{C}$ en invernadero, se presenta una diferencia de $8.2^{\circ} \mathrm{C}$ registrando los mayores valores en este último ambiente. Estos valores indican que dentro del invernadero las fluctuaciones de temperatura son más marcadas que en campo abierto e influyeron negativamente en el cultivo de romero.

Adicional a esto, es posible que el invernadero haya tenido un efecto en la calidad y/o cantidad de luz, afectando el crecimiento de las plantas de romero. Respecto a esto Kittas y Baille (1998) explican que las propiedades ópticas de los plásticos de invernadero pueden modificar el espectro de radiación solar externo teniendo como consecuencia cambios en el crecimiento y arquitectura de la planta. Teniendo en cuenta que hay una disminución en la radiación solar al usar cubiertas plásticas tradicionales (Ibrahim y Jaafar, 2011, Espí, 2012) es posible que el efecto en las plantas de romero haya sido un alargamiento de entrenudos y por lo tanto de los tallos resultando en un mayor porcentaje de tallos de categoría extra en relación a campo abierto. En geranio (Anethum graveolens L.) se ha reportado que al incrementar la radiación roja lejana se induce a la elongación de los entrenudos (Sangwang et al., 2001).

\section{CONCLUSIONES}

De manera general y bajo las condiciones de este estudio, se observó que a medida que aumentó la densidad de siembra fue mayor el rendimiento del cultivo de romero, destacándose aquella correspondiente a un espaciamiento entre plantas de $30 \times 30 \mathrm{~cm}$.

En relación al rendimiento del cultivo, medido a través de la biomasa fresca y seca, y a la calidad de los tallos de las categorías nacional y exportación, los mejores resultados se obtuvieron para el romero sembrado en el ambiente de campo abierto.

En cuanto a la calidad de los tallos es de resaltar que el cultivar Israelí presentó la mayor biomasa fresca para aquellos que fueron clasificados en la categoría extra (longitud mayor a $20 \mathrm{~cm}$ ). Así mismo, el ambiente del invernadero favoreció una mayor producción de tallos de esta calidad.

Respecto al comportamiento productivo de los cultivares, es de resaltar que el rendimiento expresado en biomasa seca fue superior en el romero lsraelí sembrado en campo abierto. En la producción de tallos calidad nacional (longitud $<14.9 \mathrm{~cm}$ ), se destacó el romero Crespo. El cultivar Israelí produjo la mayor cantidad de tallos calidad exportación en campo abierto, mientras que en el invernadero fue superior el Crespo. 
Debido a que los mejores resultados relacionados con el rendimiento y la calidad se obtuvieron para el romero plantado a la mayor densidad de siembra evaluada, se sugiere realizar en estudios posteriores la evaluación de densidades de siembra mayores a ésta.

\section{AGRADECIMIENTOS}

El presente trabajo hace parte de los resultados del proyecto titulado: "Efecto de la densidad de siembra y el ambiente de cultivo sobre la productividad, la cantidad y calidad de aceites esenciales en materiales de romero (Rosmarinus officinalis)" cofinanciado por Colciencias (112352128892 de 2011) y ejecutado por la Universidad Militar Nueva Granada a través del proyecto de CIAS 913.

En la mayoría de los casos donde se presentaron diferencias entre los ambientes de cultivo probados, el campo abierto registró los mayores valores de rendimiento y calidad, excepto para la categoría extra que fue mayor en el invernadero.

\section{BIBLIOGRAFÍA}

1. Alarcón J. 2011. Plantas aromáticas y medicinales. Enfermedades de importancia y sus usos terapéuticos. Medidas para temporada invernal. ICA, 18-19.

2. Al-Ramamneh. 2009. Plant growth strategies of Thymus vulgaris $L$. in response to population density. Industrial crops and products, 30: 389-394.

3. Al-Sereiti M.R, Abu-Amer K.M, Sen P. 1999. Pharmacology of Rosemary (Rosmarinus officinalis Linn.) and its therapeutic potentials. Indian Journal of Experimental Biology, 37: 124-130.

4. Aphalo P.J, Ballare C.L. 1995. On the importance of information-acquiring systems in plant-plant interactions. British Ecological Society, 9: 5-14.

5. Ávila-Sosa R, Navarro-Cruz A.R, Vera-López O, Dávila-Márquez R.M, Melgoza-Palma N, Meza-Pluma R. 2011. Romero (Rosmarinus officinalis L.) una revision de sus usos no culinarios. Revista Ciencia y Mar, 43: 23-36.

6. Ballaré CL, Casal JJ, Kendrick RE. 1991. Responses of light-grown wild type and long-hypocotyl mutant cucumber seedlings to natural and stimulated shade light. Photochemestry and Photobiology, 54: 819-826.

7. Ballaré C, Scopel A, Jordan E, Vierstra R. 1994. Signaling among neighboring plants and the development of size inequalities in plant populations. Proc. Natl. Acad. Sci, 91: 10094-10098.

8. Baloch A.W, Soomro A.M, Javed M. A, Ahmed M, Bughio H.R, Mastoi N. 2002. 
Optimum plant density for high yield in rice (Oryza sativa L.). Asian Journal of Plant Sciences, 1(1): 25-27.

9. Bareño P. 2006. Hierbas aromáticas culinarias para exportación en fresco manejo agronómico, producción y costos. En: Clavijo J. Bareño P, Guido C, Chaparro L. (Eds.), p. 65-72. Últimas tendencias en hierbas aromáticas para exportación en fresco. Curso de Extensión. Facultad de Agronomía,Universidad Nacional de Colombia, sede Bogotá, Colombia, 206p.

10. Bonilla CR, Martínez F. 2010. Romero (Rosmarinus officinalis L.) Producción y manejo poscosecha. Colombia. Corredor Tecnológico Agroindustrial, Cámara de Comercio de Bogotá. 102p.

11. Chang X, Alderson P, Wright C. 2008. Solar irradiance level alters the growth of basil (Ocimum basilicum L.) and its content of volatile oils. Enviromental and Experimental Botany, 63: 216-223.

12. Cracker L. 2007. Medicinal and Aromatic Plants-Future Opportunities. Issues in new crops and new uses, 248-257.

13. Dudley S, Schmitt J. 1996. Testing the adaptive plasticity hypothesis: density-dependent selection on manipulated stem length in impatiens capensis. The American Naturalist, 147 (3): 445-465.

14. Espí E. 2012. Materiales de cubierta para invernaderos. Cuadernos de estudios agroalimentarios, 71-88p.

15. Ferreira $V$, de Almeida L, da S. Feijó E, da C. Silva D, de Oliveira R, Mielke M, do B. Costa L. 2013. Light intensity on growth, leaf micromorphology and essential oil production of Ocinum gratissimum. Revista Brasileira de Farmacognosia, 23(3): 419-424.

16. Genena A. K, Hense H, Smania Junior A, de Souza S.M. 2008. Rosemary (Rosmarinus officinalis $\mathrm{L}$ ) - a study of the composition, antioxidant and antimicrobial activities of extracts obtained with supercritical carbon dioxide. Ciencia e Tecnologia de Alimentos 28 (2): 463-469.

17. Gomes P.A, Ferreira M, de Souza I, Oliveira W, Silva L, Ronie E. 2009. Influencia do sombreamento na producao de biomassa, óleo essencial e quantidade de tricomas glandulares em cidrao (Lippia citriodora Lam.). Biotemas, 22 (4): 9-14.

18. González-Michel A. 2013. Guía técnica del cultivo de romero (Rosmarinus officinalis) Centro de Investigaciones Biológicas del Noroeste, S.C. La Paz, Baja California Sur, México. 72 p.

19. Gutierrez I.C, Guarín S. Rodríguez L.E. 2007. Prospección y evaluación de especies con potencial de follaje para la elaboración de bouquets. Agronomía Colombiana 25(1):176-188.

20. Hernández-Hernández E, Ponce-Alquicira E, Jaramillo-Flores M, Guerrero Legarreta I. 2009. Antioxidant effect of rosemary (Rosmarinus officinalis L.) and oregano (Origanum vulgare L.) extracts on TBARS and colour od model raw pork batters. Journal of Meat Science, 81: 410-417.

21. Ibrahim M, Jaafar H. 2011. Photosynthetic capacity, photochemical efficiency and chlorophyll content of three varieties of Labisia pumila Benth. exposed to open field and greenhouse growing conditions. Acta Physiol. Plant, 33: 2179-2185 
22. Katerinopoulos H.E, Pagona G, Afratis A, Stratigaskis N, Roditakis N. 2005. Composition and insect attracting activity of the essential oil of Rosmarinus officinalis. Journal of Chemical Ecology, 31 (1): 111- 122.

23. Khorshidi J, Fakhr M, Omidbaigi R, Sefidkon F. 2009. Effect of densities of planting on yield and essential oil components of fennel (Foeniculum vulgare Mill. Var. Soroksary). Journal of Agricultural Science, 1(1): 152-157.

24. Kittas C, Baille A. 1998. Determination of the spectral properties of several greenhouse cover materials and evaluation of specific parameters related to plant response. Journal of Agricultural Engineering Research, 71: 193-202.

25. Lambers H, Chapin F.S, Pons T.L. 2008. Plant Phisiological ecology. New York, USA: Springer, $604 \mathrm{p}$.

26. Li Y, Craker LE, Potter T. 1996. Effect of light level on essential oil production of sage (Salvia officinalis) and thyme (Thymus vulgaris). Acta Horticulturae. International Symposium on Medicinal and Aromatic Plants, ISHS, 426: 419 - 426.

27. Li S, Kurata K, Takakura T. 2000. Direct solar radiation penetration into row crop canopies in a lean-to greenhouse. Agricultural and Forest Meteorology, 100: 243-253.

28. Luis J, Martín Pérez R, Valdés González F. 2007. UV-B radiation effects on foliar concentrations of rosmarinic and carnosic acids in rosemary plants. Food Chemestry, 101: 1211-1215.

29. Maganga A. 2004. Influence of variety and organic cultural practices on yield and essential oil content of lavender and rosemary in interior BC. South Thompson Organic Producers Association (STOPA). 23 p.

30. Marin M, Koko $V$, Duletic-Lausevic, Marin $P_{\imath}$ Rancic D, Dajic-Stevanovic. 2006. Glandular trichomes on the leaves of Rosmarinus officinalis: morphology, stereology and histochemestry. South African Journal of Botany, 72: 378-382.

31. Morelli G, Ruberti I. 2002. Light and shade in the photocontrol of Arabidopsis growth. Trends in plant science 7(9): 399-404.

32. Moreno S, Ojeda A.M, Gaya M, Barni M.V, Castro O, van Baren C. 2012. Rosemary compounds as nutraceutical health products, Food Additive, Prof. Yehia El-Samragy (Ed.), In Tech, 157-174 p.

33. Naghdi B, Yazdani D, Ali S, Nazari F. 2004. Effects of spacing and harvesting time on herbage yield and quality/quantity of oil in thyme, Thymus vulgaris L. Insutrsial crops and products, 19: 231-236.

34. Peng Y, Yuan J, Liu F, Ye J. 2005. Determination of active components in Rosemary by capillary electrophoresis with electrochemical detection. Journal of Pharmaceutical and biomedical analysis, 39: 431-437.

35. Pierik R, Cuppens M, Voesenek L, Visser E. 2004. Interactions between ethylene and gibberellins in phytochrome -mediated shade avoidance responses in tobacco. Plant Physiology, 136: 2928-2936.

36. Porte A, de Oliveira R. L. 2001. Alecrim (Rosmarinus officinalis $L$ ): propiedades antimicrobiana e química do óleo essencial. B. CEPPA 19(2): 193-210.

37. Roselló J.A, Cosín R, Boscaiu M, Vicente O, Martínez I, Soriano P. 2006. Intragenomic diversity 
and phylogenetic systematics of wild rosemaries (Rosmarinus officinalis L. s. I., Lamiaceae) assessed by nuclear ribosomal DNA sequences (ITS). Plant Systematics and Evolution, 262: 1-12.

38. Sadeghi S, Rahnavard A, Ashrafi Z. 2009. Study importance of sowing date and plant density effect on black cumin (Cuminum carvi) yield. Botany Research International 2(2): 94-98.

39. Sangwan N.S, Farooqi A.H.A, Shabih F, Sangwan R.S. 2001. Regulation of essential oil production in plants. Plant Growth Regulation, 34: 3-21.

40. Sasikumar B. 2004.Rosemary. In Handbook of herbs and spices vol 1(Ed) Peter K.V. Woodhead Publishing Ltd, Abington, 243-255p.

41. Socaci S, Tofana M, Socaciu C. 2008. GC-MS analysis of rosemary essential oil. Bulletin UASVM, 65(2): 405-409.

42. Souza C, Schiavetto I.A., Thomazini F., Oliveira W. 2008 Processing of Rosmarinus officinalis linne extract on spray and spouted bed dryers. Brazilian Journal of Chemical Engineering, 25 (1): 59-69

43. Soto R, Vega G, Tamajón A. 2003. Efecto de diferentes densidades de plantación en Cymbopogon citratus Stapf. Revista Cubana de plantas medicinales, 8 (2): Disponible en: http://scielo.sld.cu/ scielo.php?script=sci_arttext\&pid=S102847962003000200008\&lng=es

44. Taiz L, Zeiger E. 2010. Plant Phisiology $5^{a}$ edition. Sinauer Associates, Sunderland, Massachusetts, USA. 177, 385-387p.

45. Viuda-Martos M, Ruiz-Navajas $Y$, FernándezLópez J, Pérez-Álvarez J.A, 2007. Chemical composition of the essential oils obtained from some spices widely used in Mediterranean region. Acta Chimica Slovenica, 54: 921-926.

46. Xiao C, Dai H, Liu H, Wang Y, Tang H. 2008. Revealing the metabonomic variation of Rosemary extracts using H NMR spectroscopy and multivariate data analysis. Journal of Agricultural and Food Chemestry, 56: 10142-10153.

47. Yanishlieva-Maslarova N.V, Heinonen I.M. 2001. Rosemary and sage as antioxidants. In Handbook of herbs and spices, vol 1(Ed) Peter K.V. Woodhead Publishing Ltd, Abington, 269-275p.

48. Zaouali Y, Bouzaine T, Boussaid M. 2010. Essential oils composition in two Rosmarinus officinalis $L$. varieties and incidence for antimicrobial and antioxidant activities. Food and Chemical Toxicology, 48: 3144-3152. 